\title{
Discovery of an H I-rich Gas Reservoir in the Outskirts of SZ-effect-selected Clusters
}

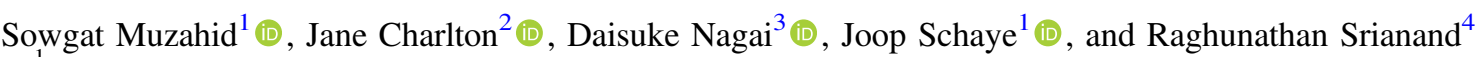 \\ ${ }^{1}$ Leiden Observatory, Leiden University, P.O. Box 9513, NL-2300 RA Leiden, The Netherlands; sowgat@strw.leidenuniv.nl \\ ${ }^{2}$ Department of Astronomy \& Astrophysics, The Pennsylvania State University, State College, PA 16801, USA \\ ${ }^{3}$ Department of Physics, Yale University, New Haven, CT 06520, USA \\ ${ }^{4}$ Inter-University Centre for Astronomy and Astrophysics, Post Bag 4, Ganeshkhind, Pune 411 007, India \\ Received 2017 July 26; accepted 2017 August 8; published 2017 August 29
}

\begin{abstract}
We report on the detection of three strong $\mathrm{H}$ I absorbers originating in the outskirts (i.e., impact parameter, $\rho_{\mathrm{cl}} \approx$ (1.6-4.7) $\left.r_{500}\right)$ of three massive $\left(M_{500} \sim 3 \times 10^{14} M_{\odot}\right)$ clusters of galaxies at redshift $z_{\mathrm{cl}} \approx 0.46$, in the Hubble Space Telescope Cosmic Origins Spectrograph (HST/COS) spectra of three background UV-bright quasars. These clusters were discovered by the $2500 \mathrm{deg}^{2}$ South Pole Telescope Sunyaev-Zel'dovich (SZ) effect survey. All three COS spectra show a partial Lyman limit absorber with $N(\mathrm{HI})>10^{16.5} \mathrm{~cm}^{-2}$ near the photometric redshifts $|\Delta z /(1+z)| \approx 0.03)$ of the clusters. The compound probability of the random occurrence of all three absorbers is $<0.02 \%$, indicating that the absorbers are most likely related to the targeted clusters. We find that the outskirts of these SZ-selected clusters are remarkably rich in cool gas compared to existing observations of other clusters in the literature. The effective Doppler parameters of the Lyman series lines, obtained using a single-cloud curve-ofgrowth (COG) analysis, suggest a nonthermal/turbulent velocity of a few $\times 10 \mathrm{~km} \mathrm{~s}^{-1}$ in the absorbing gas. We emphasize the need for uniform galaxy surveys around these fields and for more UV observations of quasar-cluster pairs in general in order to improve the statistics and gain further insights into the unexplored territory of the largest collapsed cosmic structures.
\end{abstract}

Key words: galaxies: clusters: intracluster medium - galaxies: halos - intergalactic medium - quasars: absorption lines

\section{Introduction}

Galaxy clusters are the most massive gravitationally bound structures in the universe. With 100-1000 galaxies and total masses of $\sim 10^{14-15} M_{\odot}$, gas accreting onto a cluster is typically heated to a very high temperature. In fact, X-ray observations have revealed enormous quantities of diffuse, hot $\left(\sim 10^{7-8} \mathrm{~K}\right)$ gas in the central regions of galaxy clusters within which the mean mass density is over 500 times the critical density of the universe (i.e., $<r_{500}$; see Voit 2005 for a review). The origin of the energy radiated away via X-rays, which have been the main source of information on the intracluster medium (ICM) so far, is thermal bremsstrahlung with power $P \propto n_{e}^{2} T_{e}^{1 / 2}$. However, the ICM in cluster outskirts (i.e., $\left.\rho_{\mathrm{cl}} \sim(1-5) r_{500}\right)$, where the density and temperature are considerably lower than in the core, is not bright enough to detect in X-ray emission. Consequently, the outskirts of galaxy clusters, particularly at high $-z$, are not well explored observationally. This is partly because of the lack of sensitive diagnostics for probing the cool/warm-hot gas, with $T \sim 10^{4-6} \mathrm{~K}$, that prevails in the circumcluster medium (CCM).

In recent years, with the advent of high-resolution cosmological simulations and deep X-ray observations of a handful of nearby clusters, cluster outskirts have emerged as one of the new frontiers of study in cluster astrophysics and cosmology (e.g., Simionescu et al. 2011; Walker et al. 2012; Urban et al. 2014; Lau et al. 2015; Bahé et al. 2017). This environment is the interface between clusters and the cosmic web. In the outskirts, galaxies and groups of galaxies are stripped of their metal-rich gas by tidal forces and by the ram pressure provided by the cluster, enriching the ICM with heavy elements. The outskirts of galaxy clusters may harbor a substantial fraction of the "missing baryons" (e.g., Gonzalez et al. 2007, 2013), which could reside in the cool/warm-hot
$\left(T \sim 10^{4-6} \mathrm{~K}\right)$ gas phase. Probing the CCM is thus crucial for understanding gas flows, metal enrichment history, and the baryon budget in the largest collapsed environments.

Since cluster outskirts are beyond the reach of present-day $\mathrm{X}$-ray telescopes, an effective alternative is to use absorption line spectroscopy of background UV-bright quasars to probe the CCM. This technique has provided a wealth of information regarding the circumgalactic medium (CGM) of both low- and high-z galaxies (e.g., Turner et al. 2014; Werk et al. 2014; Kacprzak et al. 2015). However, except for a very few studies (i.e., Yoon et al. 2012; Burchett et al. 2017; Yoon \& Putman 2017), it has not yet been used to probe the CCM. Yoon et al. (2012) have studied 43 Ly $\alpha$ absorbers along 23 background quasar (QSO) sightlines toward the Virgo cluster using Cosmic Origins Spectrograph (COS), Space Telescope Imaging Spectrograph (STIS), and Goddard High-Resolution Spectrograph (GHRS) data. Interestingly, they found that the cool gas in Virgo is preferentially located in the cluster outskirts and is associated with substructures. Recently, Burchett et al. (2017) have studied the CCM of seven X-ray detected clusters with masses of $M_{200} \sim$ few $\times 10^{14} M_{\odot}$. Their sightlines typically pass within $300 \mathrm{kpc}$ of a cluster galaxy. They have reported a very low covering fraction $(\approx 18 \%)$ of $\mathrm{HI}$ absorbing gas (equivalent width $>30 \mathrm{~m} \AA$ ) in the CGM of cluster galaxies as compared to field/group galaxies $(\approx 80 \%-100 \%)$.

Motivated by the lack of UV observations of the CCM and its importance, we have built a sample of QSO-cluster pairs by cross-correlating the Sunyaev-Zel'dovich (SZ) effect selected cluster catalog of Bleem et al. (2015) and the all-sky UV-bright QSO catalog (UVQS) of Monroe et al. (2016). As a pilot program we have obtained far-UV (FUV) spectra of three quasars using the Hubble Space Telescope (HST)/COS. These quasars probe the outskirts of three SZ-selected clusters of 
Table 1

Details of the QSO-Cluster Pairs

\begin{tabular}{|c|c|c|c|c|c|c|c|c|c|c|c|c|}
\hline $\begin{array}{l}\text { Cluster } \\
\text { (1) }\end{array}$ & $\begin{array}{l}\text { R.A.cl } \\
\text { (J2000) } \\
\text { (2) }\end{array}$ & $\begin{array}{l}\text { Decl.cl } \\
\text { (J2000) } \\
\text { (3) }\end{array}$ & $\begin{array}{l}z_{\mathrm{cl}} \\
(4)\end{array}$ & $\begin{array}{c}M_{500} \\
\left(10^{14} M_{\odot}\right) \\
(5)\end{array}$ & $\begin{array}{c}r_{500} \\
(\mathrm{Mpc}) \\
(6)\end{array}$ & $\begin{array}{l}\text { QSO } \\
\text { (7) }\end{array}$ & $\begin{array}{l}z_{\text {qso }} \\
(8)\end{array}$ & $\begin{array}{l}\text { FUV } \\
\text { (9) }\end{array}$ & $\begin{array}{c}\rho_{\mathrm{cl}} \\
(\mathrm{Mpc}) \\
(10)\end{array}$ & $\begin{array}{c}\rho_{\mathrm{cl}} / r_{500} \\
(11)\end{array}$ & $\begin{array}{l}z_{\mathrm{abs}} \\
(12)\end{array}$ & $\begin{array}{c}\log N(\mathrm{H} \mathrm{I}) \\
\left(N / \mathrm{cm}^{-2}\right) \\
(13)\end{array}$ \\
\hline J0041-5107 & 10.2932 & -51.1286 & $0.45 \pm 0.04$ & $3.04 \pm 0.87$ & 0.87 & J0040-5057 & 0.608 & 17.43 & 3.80 & 4.4 & 0.43737 & $18.63 \pm 0.07$ \\
\hline J2016-4517 & 304.0050 & -45.2978 & $0.45 \pm 0.03$ & $3.19 \pm 0.89$ & 0.89 & J2017-4516 & 0.692 & 17.81 & 4.20 & 4.7 & 0.43968 & $16.52 \pm 0.05$ \\
\hline J2109-5040 & 317.3825 & -50.6765 & $0.47 \pm 0.04$ & $3.81 \pm 0.87$ & 0.93 & $\mathrm{~J} 2109-5042$ & 1.262 & 17.93 & 1.47 & 1.6 & 0.51484 & $16.68 \pm 0.03$ \\
\hline
\end{tabular}

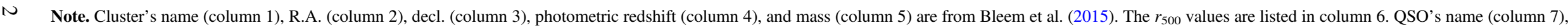

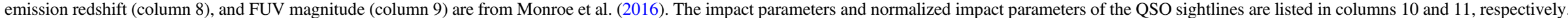

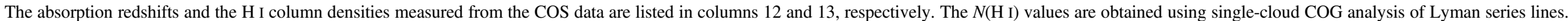

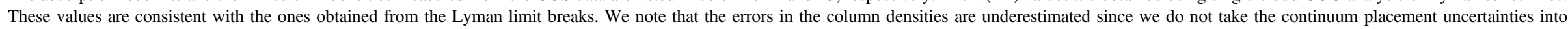
account. A more realistic error would be $0.10-0.15$ dex. 
masses $M_{500} \sim 3 \times 10^{14} M_{\odot}$ at redshift $z_{\mathrm{cl}} \approx 0.46$ with impact parameters, $\rho_{\mathrm{cl}}$, of $1.5-4.2 \mathrm{Mpc}\left(\rho_{\mathrm{cl}} / r_{500} \approx 1.6-4.7\right)$. The details of the QSO-cluster pairs are listed in Table 1. Intriguingly, in all three cases, we detect strong $\mathrm{H}$ I absorption with $N(\mathrm{H} \mathrm{I})>10^{16.5} \mathrm{~cm}^{-2}$ at the redshifts of the foreground clusters.

This Letter is organized as follows. In Section 2, we describe our COS observations. The analysis and the main results are presented in Section 3. The possible implications of our observations are discussed in Section 4. Throughout the Letter we adopt a flat $\Lambda \mathrm{CDM}$ cosmology with $H_{0}=71$ $\mathrm{km} \mathrm{s}^{-1} \mathrm{Mpc}^{-1}, \Omega_{\mathrm{M}}=0.3$, and $\Omega_{\Lambda}=0.7$. All the distances given are proper (physical) distances.

\section{Observations}

UV spectra of the three background UV-bright (FUV $<18$ ) quasars were obtained using $H S T / C O S$ Cycle-24 observations under program ID: GO-14655 (PI: Muzahid). The properties of COS and its in-flight operations are discussed in Osterman et al. (2011) and Green et al. (2012). The observations consist of G130M and G160M FUV grating integrations covering 1100-1800 $\AA$ at a medium resolution of $R \sim 18,000$. $^{5}$ The data were retrieved from the $H S T$ archive and reduced using the STScI CalCOS v3.1.8 pipeline software. The reduced, fluxcalibrated individual exposures were aligned and coadded using the IDL code "coadd_x1d"(v3.1) developed by Danforth et al. (2010). The combined spectra have a signal-to-noise ratio $(\mathrm{S} / \mathrm{N})$ of 5-10 per resolution element. Each combined spectrum was binned by 3 pixels as the COS FUV spectra, with 6 raw pixels per resolution element, are highly oversampled. The analysis/results presented here are not affected by this re-binning. Continuum normalizations were done by fitting the line-free regions with smooth low-order polynomials.

\section{Analysis and Results}

In this section, we will first describe the properties of the absorbers and the targeted clusters. Next, the newly obtained data will be compared with those in the literature.

1. The $z_{a b s}=0.43737$ system toward UVQS J0040-5057. The absorber has a systemic velocity of $\approx-2600 \mathrm{~km} \mathrm{~s}^{-1}$ with respect to the photometric redshift $(0.45 \pm 0.04)$ of the cluster J0041-5107 (Bleem et al. 2015). This velocity is well within the $1 \sigma$ uncertainty of the cluster redshift, i.e., $|\Delta z /(1+z)| \approx 0.03 \approx 9000 \mathrm{~km} \mathrm{~s}^{-1}$. This is the strongest $\mathrm{H}$ I absorber among the three systems studied here, producing a full H I Lyman limit break at $\approx 1315 \AA$ (see the top panel of Figure 1). The full break allows us to estimate a lower limit on $N(\mathrm{HI})^{6}$ of $10^{17.7} \mathrm{~cm}^{-2}$ assuming the flux below $1315 \AA$ to be less than $10^{-16} \mathrm{erg} \mathrm{cm}^{-2} \mathrm{~s}^{-1} \AA^{-1}$. A single-component curveof-growth (COG) analysis of all the unblended Lyman series lines yields a column density of $10^{18.63 \pm 0.07} \mathrm{~cm}^{-2}$ and an effective Doppler parameter of $b_{\mathrm{eff}}=41 \pm 1 \mathrm{~km} \mathrm{~s}^{-1}$ (see Figure 2). The mass of the corresponding cluster is $M_{500}=(3.04 \pm 0.87) \times 10^{14}$ $M_{\odot}$, corresponding to $r_{500}=0.87 \mathrm{Mpc}$ (see Table 1 ). The impact parameter of $3.80 \mathrm{Mpc}$ gives $\rho_{\mathrm{cl}} / r_{500} \approx 4.4$.

\footnotetext{
5 No flux is observed below $1315 \AA$ in the spectrum of QSO UVQS J0040-5057 due to the strong Lyman limit break in the spectrum caused by the $z_{\mathrm{abs}}=0.43737$ absorber studied here.

$6 N(\mathrm{H} \mathrm{I})=\frac{\tau_{\mathrm{LL}}}{\sigma}{ }_{\mathrm{HI}}$, where $\sigma_{\mathrm{HI}} \approx 6.3 \times 10^{-18} \mathrm{~cm}^{2}$ is the $\mathrm{H} \mathrm{I}$ photoionization cross-section and $\tau_{\mathrm{LL}}=-\ln \left(\frac{I}{I_{0}}\right)$ is the optical depth at the Lyman limit.
}

2. The $z_{a b s}=0.43968$ system toward UVQS J2017-4516. The systemic velocity of the absorber with respect to the photometric redshift of the corresponding cluster $(\mathrm{J} 2016-4517)$ is $\approx-2100$ $\mathrm{km} \mathrm{s}^{-1}$, which is well within the $1 \sigma$ uncertainty $\| \Delta z /(1+$ $z) \mid \approx 0.02 \approx 6000 \mathrm{~km} \mathrm{~s}^{-1}$ ) of the cluster's photometric redshift (Bleem et al. 2015). The absorber exhibits a partial break at the H I Lyman limit corresponding to $N(\mathrm{H} \mathrm{I}) \approx 10^{16.6} \mathrm{~cm}^{-2}$ (see the middle panel of Figure 1). A single-component COG analysis of all the unblended Lyman series lines gives a consistent $N(\mathrm{HI})$ of $10^{16.52 \pm 0.05} \mathrm{~cm}^{-2}$ and $b_{\text {eff }}$ of $25 \pm 1 \mathrm{~km} \mathrm{~s}^{-1}$ (see Figure 2). The mass and radius of the cluster are $M_{500}=(3.19 \pm 0.89) \times 10^{14}$ $M_{\odot}$ and $r_{500}=0.89 \mathrm{Mpc}$, respectively. The impact parameter of $4.20 \mathrm{Mpc}$ corresponds to $\rho_{\mathrm{cl}} / r_{500} \approx 4.7$.

3. The $z_{a b s}=0.51484$ system toward UVQS J2109-5042. This absorber shows a somewhat larger systemic velocity of $\approx+9000 \mathrm{~km} \mathrm{~s}^{-1}$ with respect to the cluster J2109-5040. Such a velocity, however, is consistent within the $1 \sigma$ uncertainty of the cluster's photometric redshift, i.e., $|\Delta z /(1+z)| \approx 0.03 \approx 9000$ $\mathrm{km} \mathrm{s}^{-1}$ (Bleem et al. 2015). The partial break seen in the COS spectrum (see the bottom panel of Figure 1 ) gives $N(\mathrm{HI}) \approx$ $10^{16.8} \mathrm{~cm}^{-2}$, which is consistent with the value we obtain from the COG analysis of the unblended Lyman series lines (i.e., $10^{16.68 \pm 0.03} \mathrm{~cm}^{-2}$; see Figure 2). We obtain $b_{\text {eff }}$ of $32 \pm$ $1 \mathrm{~km} \mathrm{~s}^{-1}$ from the COG analysis. The mass of the cluster, $M_{500}=(3.81 \pm 0.87) \times 10^{14} M_{\odot}, \quad$ corresponds to $r_{500}=$ $0.93 \mathrm{Mpc}$. The impact parameter of the cluster is $1.5 \mathrm{Mpc}$ so that $\rho_{\mathrm{cl}} / r_{500} \approx 1.6$.

In Figure 3, we show the clustocentric radial profile of $N$ (H I), combining our measurements with the handful of studies that exist in the literature. The data points corresponding to the Virgo and Coma clusters are taken from Yoon et al. (2012) and Yoon \& Putman (2017). We have used the publicly available code massconvert (Hu \& Kravtsov 2003), which assumes a Navarro-Frenk-White (NFW) density profile, to convert the $M_{200}$ values of Coma $\left(1.4 \times 10^{15} M_{\odot}\right)$ and Virgo $(2.2 \times$ $\left.10^{14} M_{\odot}\right)$ as given in Yoon \& Putman (2017) to $M_{500}\left(r_{500}\right)$. The impact parameters of the quasar sightlines with respect to Virgo, which are not explicitly given in Yoon et al. (2012), are calculated assuming the center of the cluster to be the center of Cluster-A containing M87. Note that a velocity window of $\pm 3024 \mathrm{~km} \mathrm{~s}^{-1}$ around the systemic velocity of Coma and a velocity window of -438 to $+1862 \mathrm{~km} \mathrm{~s}^{-1}$ around the systemic velocity of Virgo were considered by the authors for connecting absorbers to the corresponding cluster. The lower bound in velocity for Virgo was affected by the Galactic Ly $\alpha$ absorption. In Figure 3, we also compare to the recent study of Burchett et al. (2017), who have presented H I column densities around seven X-ray-detected clusters in the redshift range $0.1-0.45$ and within a velocity window of $\pm 2000 \mathrm{~km} \mathrm{~s}^{-1}$. The massconvert routine is used to convert their $M_{200}$ values to $M_{500}\left(r_{500}\right)$.

The lack of any trend between $N(\mathrm{HI})$ and the normalized clustocentric impact parameter is evident from Figure 3. This is in contrast to the results of absorption line studies of the CGM (e.g., Prochaska et al. 2011; Tumlinson et al. 2013) in which an anti-correlation between $N(\mathrm{HI})$ (or the equivalent width) and impact parameter is routinely seen. Next, we note that the SZeffect-selected clusters from this study are the ones that exhibit the highest $\mathrm{H}$ I column densities in the outskirts. Only $3.7 \%$ $(2 / 54)$ of the Virgo sightlines show a partial Lyman limit system (pLLS; $N(\mathrm{HI})>10^{16.2} \mathrm{~cm}^{-2}$ ). None of the Coma sightlines show a pLLS. Note that owing to the low redshifts, 


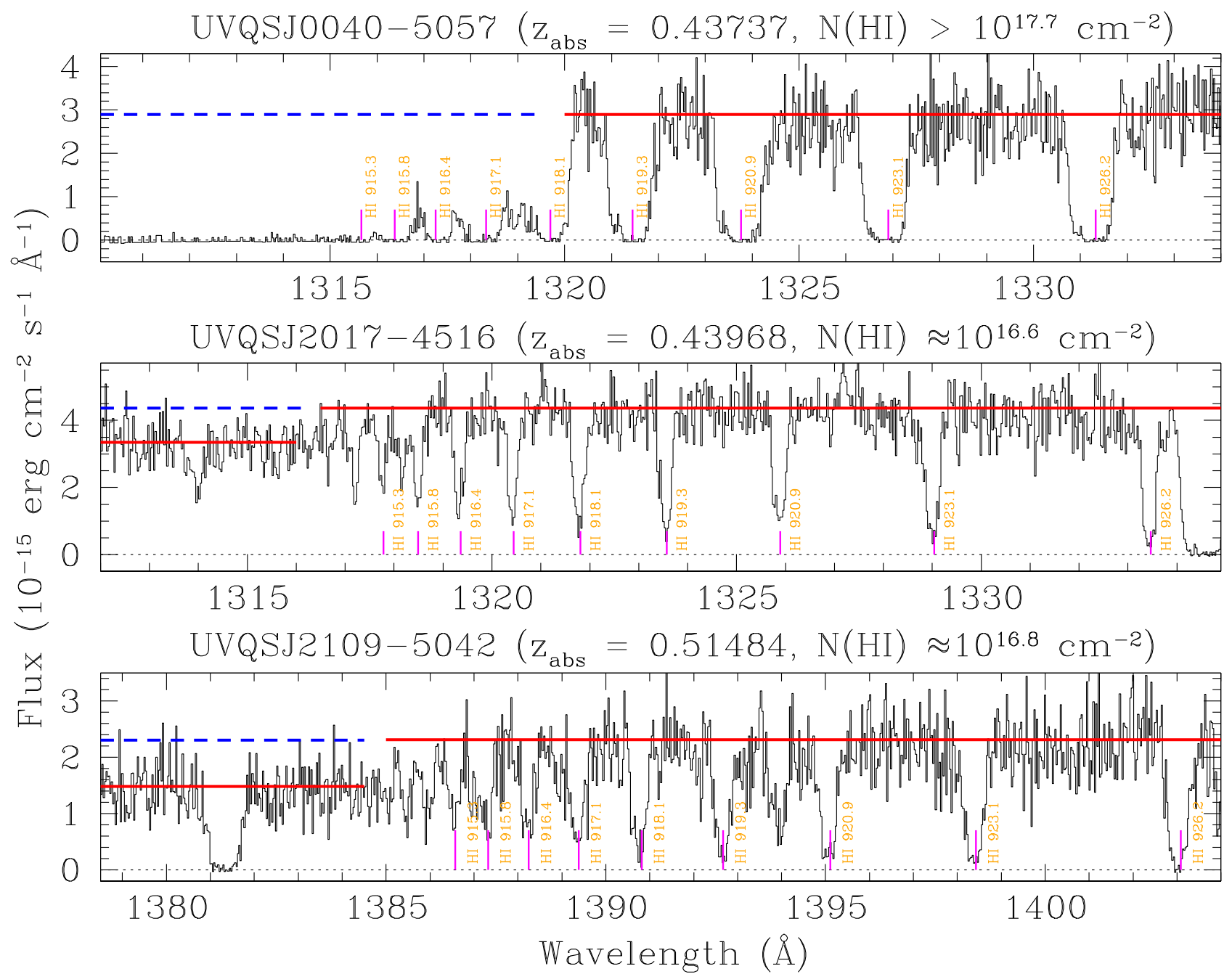

Figure 1. Selected parts of the COS spectra showing the Lyman limit breaks caused by the absorbers with redshifts consistent with the photometric redshifts of the targeted clusters. The higher-order H I Lyman series lines are marked. The horizontal (red) lines are the adopted continua. The horizontal (blue) dashed lines blueward of the Lyman limit breaks are the extrapolated continua. The full break produced by the absorber at $z_{\text {abs }}=0.43737$ toward UVQS J0040-5057 only provides a lower limit on $N(\mathrm{H} \mathrm{I})$ of $10^{17.7} \mathrm{~cm}^{-2}$. The partial breaks seen toward UVQS J2017-4516 and UVQS J2019-5042 yield $\log N(\mathrm{H} \mathrm{I}) / \mathrm{cm}^{-2} \approx 16.6$ and 16.8 , respectively. These $N(\mathrm{H} \mathrm{I})$ values are consistent with the ones obtained using single-cloud COG analysis.

the $N(\mathrm{H} \mathrm{I})$ measurements for Virgo and Coma rely only on the Ly $\alpha$ line, and the systems with $N(\mathrm{HI})>10^{14.5} \mathrm{~cm}^{-2}$ are presumably saturated. Nonetheless, even if we assume that all of the absorbers with $N(\mathrm{H} \mathrm{I})>10^{14.5} \mathrm{~cm}^{-2}$ toward Virgo and Coma are pLLS, the fraction is only $\approx 20 \pm 5 \%(14 / 71)$. None of the absorbers in the Burchett et al. (2017) sample are a pLLS. The highest $N(\mathrm{HI})$ they observed is $10^{14.93 \pm 0.03} \mathrm{~cm}^{-2}$, which is well constrained by the presence of $\operatorname{Ly} \alpha, \operatorname{Ly} \beta$, and Ly $\gamma$ lines. All other absorbers have $\log N(\mathrm{H} \mathrm{I}) / \mathrm{cm}^{-2}<14$.1. In Section 4, we return to the issue of apparent abundance of strong H I absorbers toward our targeted SZ clusters.

In Figure 4, we show the H I column density profile around low- $z$ galaxies using three different galaxy samples from the literature. The COS-Halos points represent the $N(\mathrm{HI})$ profile around isolated, bright $\left(\sim L_{*}\right)$ galaxies within $\pm 600 \mathrm{~km} \mathrm{~s}^{-1}$. For the sample of Prochaska et al. (2011) we have selected the galaxies with at least one additional $L>0.1 \mathrm{~L}_{*}$ galaxy detected within $3 \mathrm{Mpc}$ of the sightline and with a velocity offset of $|\Delta v|<400 \mathrm{~km} \mathrm{~s}^{-1}$. Therefore, these data points essentially provide the radial profile of $N(\mathrm{HI})$ around group galaxies. Here, we note that some of the COS-Halos galaxies might also have companions within $3 \mathrm{Mpc}$ and with $|\Delta v|<400 \mathrm{~km} \mathrm{~s}^{-1}$.
The points corresponding to Burchett et al. (2017) are for cluster galaxies with $\rho_{\text {galactocentric }}<300 \mathrm{kpc}$ and $|\Delta v|<$ $600 \mathrm{~km} \mathrm{~s}^{-1}$. We note that at any given impact parameter, when data are available, the field galaxies tend to have the highest column densities followed by the group galaxies. As noted by Burchett et al. (2017), a significant suppression in $N$ $(\mathrm{HI})$ is apparent for their cluster galaxies. The $N(\mathrm{H} \mathrm{I})$ measurements of the SZ clusters, as indicated by the dotted lines, are more than two orders of magnitude higher than the measurements/limits obtained by Burchett et al. (2017).

\section{Discussion and Summary}

The H I column densities we measure (i.e., $N(\mathrm{HI})>$ $\left.10^{16.5} \mathrm{~cm}^{-2}\right)$ in the outskirts $\left(\rho_{\mathrm{cl}} / r_{500}=1.6-4.7\right)$ of SZselected clusters are clearly significantly higher than the other existing measurements around X-ray-detected clusters (see Figure 3). One of the possible reasons could be that the strong $\mathrm{H} \mathrm{I}$ absorbers we detect are not related to the targeted clusters. Recall that we noticed large velocity offsets (i.e., -2600 , -2100 , and $+9000 \mathrm{~km} \mathrm{~s}^{-1}$ ), albeit consistent within the $1 \sigma$ uncertainties, between the absorber's and the cluster's redshifts. 
UVQSJ0040-5057

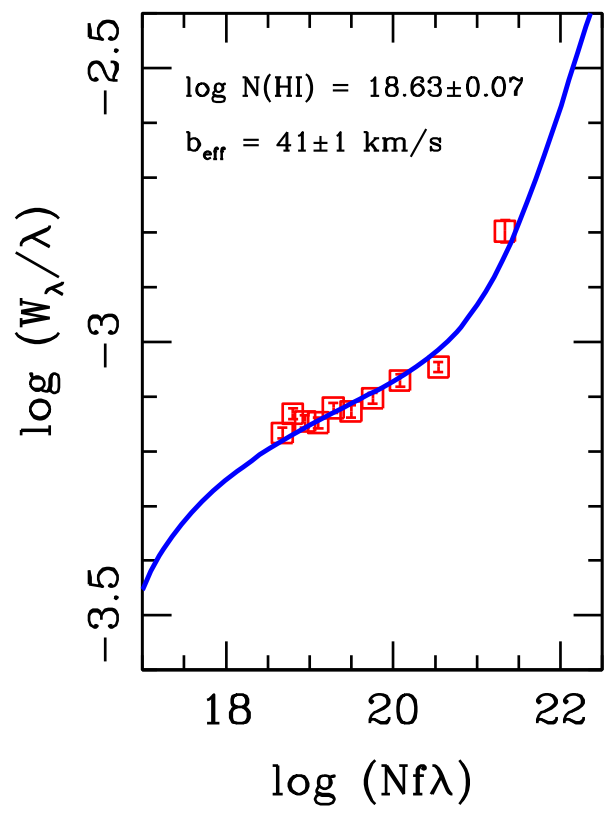

UVQSJ2017-4516

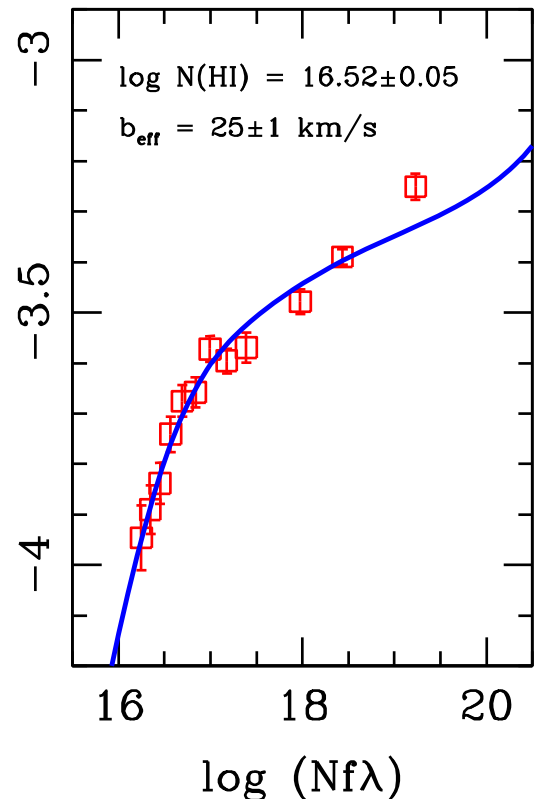

UVQSJ2109-5042

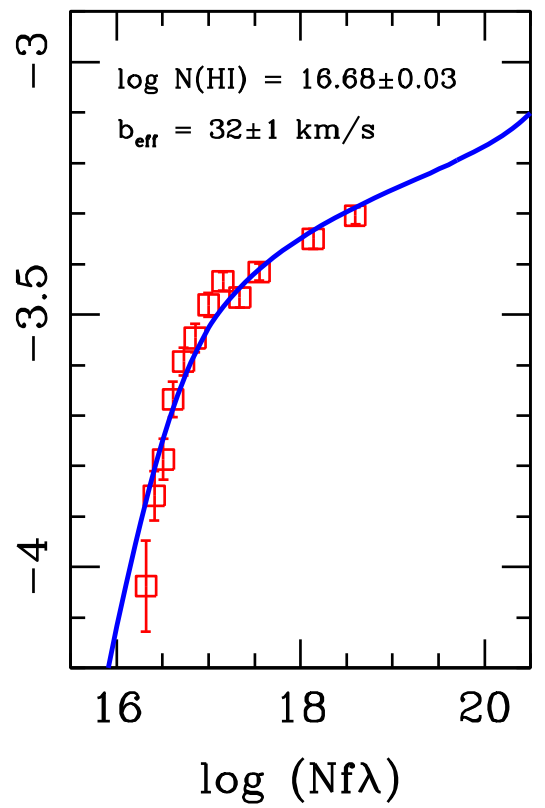

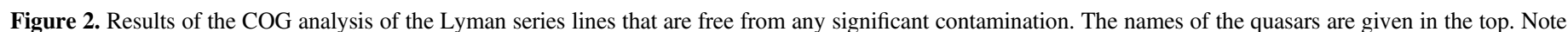

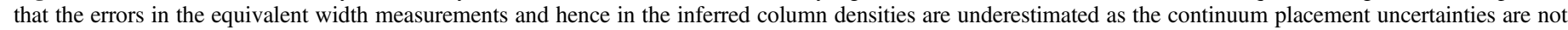

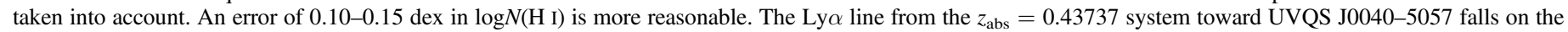
damping part of the COG. In fact, we do see a weak damping wing in the Ly $\alpha$ absorption.

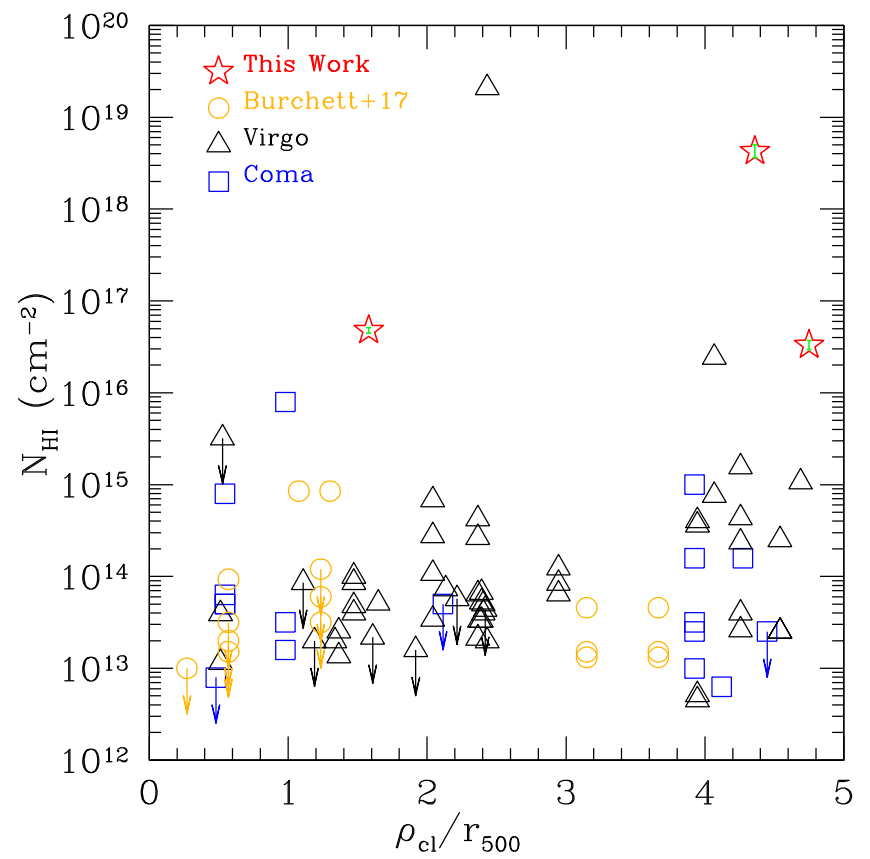

Figure 3. H I column density against the clustocentric impact parameter normalized by $r_{500}$. The data points corresponding to the Virgo and Coma clusters are from Yoon et al. (2012) and Yoon \& Putman (2017). Because no convincing Ly $\alpha$ absorption is seen within $\pm 2000 \mathrm{~km} \mathrm{~s}^{-1}$ of the cluster MaxBCG J217.847+24.683 in Burchett et al. (2017), we have assumed $N(\mathrm{H} \mathrm{I})<10^{13} \mathrm{~cm}^{-2}$, which is consistent with the error spectrum. No obvious trend is seen in the $N(\mathrm{H} \mathrm{I})$ radial profile.

We, therefore, calculate the probability of random occurrence of each absorber around the cluster redshift within the $\pm 1 \sigma$ uncertainties in the photometric redshifts (see Table 1) by using the observed $\frac{d \mathcal{N}(>N, z)}{d z}$ of low- $z$ H I absorbers (Danforth et al. 2016). The probabilities turn out be $<4.2 \%, 6.3 \%$, and $8.7 \%$

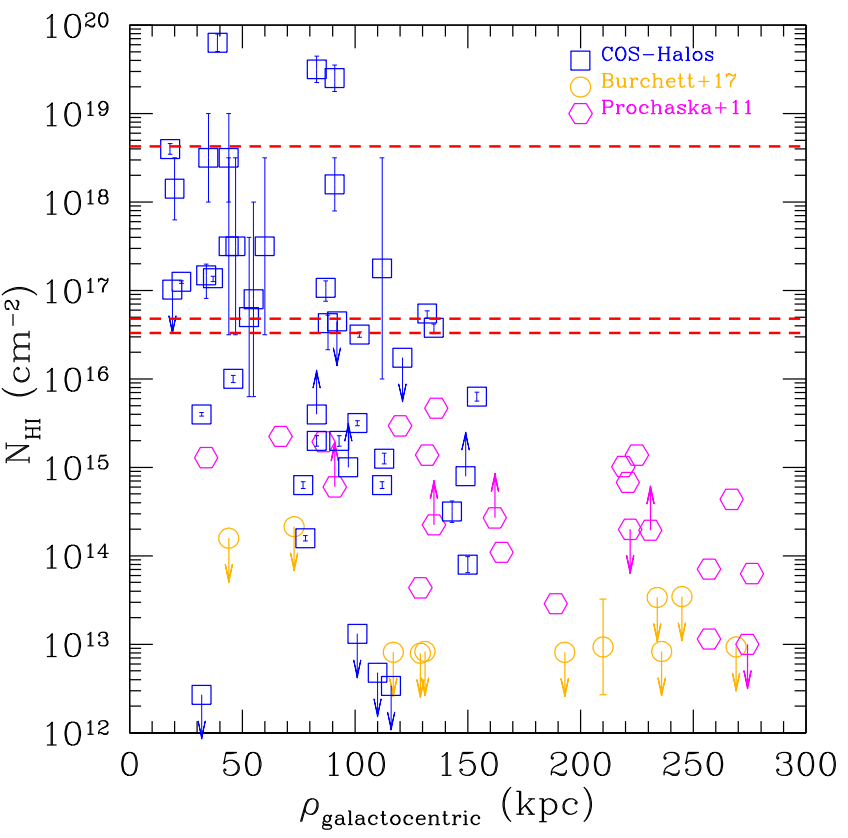

Figure 4. Radial profile of $\mathrm{H}$ I absorbing gas around galaxies. The (blue) squares represent $L \sim L_{*}$ field galaxies at $z \approx 0.2$ studied by the COS-Halos team (Werk et al. 2014; Prochaska et al. 2017). The (magenta) hexagons are from the sample of galaxies with $L>0.1 L_{*}$ from Prochaska et al. (2011). Note that for each of these galaxies at least one additional galaxy with $L>0.1 L_{*}$ has been detected within $3 \mathrm{Mpc}$ of the sightline and with $|\Delta v|<400 \mathrm{~km} \mathrm{~s}^{-1}$. The (orange) circles represent the cluster galaxies from Burchett et al. (2017). The horizontal dashed lines are the $N(\mathrm{H} \mathrm{I})$ values for the three clusters studied here.

for the absorbers toward J0040-5057, J2017-4516, and J2109-5042, respectively. Because the events are independent, the compound probability of the random occurrence of all three absorbers is $<0.02 \%$. Thus, it is unlikely that the absorbers are 
unrelated to the clusters. Nonetheless, spectroscopic confirmation of the redshifts of the clusters is of the utmost importance.

One of the interesting questions regarding the nature of the absorbers is: do they arise from the CCM or are they related to the CGM of cluster galaxies close to the lines of sight? If they are related to the CGM of $\sim L_{*}$ galaxies as in the COS-Halos survey, then it is evident from Figure 4 that the host galaxies would be detected within $150 \mathrm{kpc}$ of the QSO lines of sight. The majority of the absorbers with metal lines toward Virgo and Coma also have galaxies within $300 \mathrm{kpc}$ and $300 \mathrm{~km} \mathrm{~s}^{-1}$ (Yoon \& Putman 2013, 2017). Note that we also detect a range of metal lines (e.g., C II, C III, N III) from all of these absorbers. A faint dwarf galaxy very close to the QSO (possibly within the QSO PSF) can also produce such strong $\mathrm{H}$ I absorption. Alternatively, these absorbers could be probing stripped-off CGM material far away from the host galaxies. A uniform search for bright continuum-emitting and faint lineemitting galaxies in these fields, using facilities like the Very Large Telescope/Multi Unit Spectroscopic Explorer (VLT/ MUSE), is crucial for a better understanding of the origin(s) of the absorbers.

Our single-component COG analyses of the Lyman series lines yielded $b_{\text {eff }}$ in the range of $25-41 \mathrm{~km} \mathrm{~s}^{-1}$ (Figure 2). This corresponds to a nonthermal broadening of $21-38 \mathrm{~km} \mathrm{~s}^{-1}$ assuming the temperature of the $\mathrm{H} \mathrm{I}$ absorbing gas is $\sim 10^{4} \mathrm{~K}$. The presence of multiple (unresolved) components and/or higher gas temperature would only lessen the nonthermal contribution to the line broadening. In hydrodynamical simulations, the predicted merger-induced random gas motions are on the order a few $100 \mathrm{~km} \mathrm{~s}^{-1}$ in the outskirts of galaxy clusters (see, e.g., Figure 4 in Nagai et al. 2013). The measured $b_{\text {eff }}$ values are consistent with the ones observed in the CGM of individual low- $z$ galaxies (see, e.g., Figure 11 in Tumlinson et al. 2013), but are significantly lower than the values predicted in simulations of cluster outskirts. This suggests that the observed $\mathrm{HI}$ absorbing gas is associated with the collapsed substructures as opposed to the CCM at large.

If the absorbers stem from the CGM of cluster galaxies, then the difference of more than two orders of magnitude in $N(\mathrm{H} \mathrm{I})$ compared to the measurements/limits placed by Burchett et al. (2017) is intriguing (see Figure 4). We note that the clusters in Burchett et al. (2017) are X-ray detected and primarily at $z \lesssim 0.2$ but with masses $\left(\sim\right.$ few $\left.\times 10^{14} M_{\odot}\right)$, by and large, similar to our SZ-selected clusters at $z \approx 0.46$. It is unlikely that the redshift difference could make such a drastic difference in the observed $N(\mathrm{HI})$ values. We further note that no unambiguous $\mathrm{HI}$ absorption is detected (e.g., $N(\mathrm{HI})<$ $\left.10^{14.3} \mathrm{~cm}^{-2}\right)$ from the highest-redshift cluster galaxy $\left(z_{\text {gal }}=\right.$ 0.4560 ) toward the cluster GMBCG J255.55+64.23 in the sample of Burchett et al. (2017). This supports the idea that the redshift evolution of cluster outskirts is unlikely to be the cause. However, currently we are restricted by a very small sample. UV spectroscopic observations of many more galaxy clusters are indispensable for further advancement.

Yoon et al. (2012) have noticed that the sightlines passing through substructures in the periphery of Virgo are likely to have higher Ly $\alpha$ equivalent widths. The same scenario might be true for the systems studied here. In fact, a detection of a metal-poor $([\mathrm{O} / \mathrm{H}]=-1.6)$, sub-damped Ly $\alpha$ absorber (subDLA; $\log N(\mathrm{H} \mathrm{I}) / \mathrm{cm}^{-2} \approx 19.3$ ) has been reported by Tripp et al. (2005) in the outskirts of Virgo near the NGC 4261 galaxy group (see Figure 3). Interestingly, no bright galaxy with a small impact parameter has been found by the authors. The nearest known sub- $L_{*}$ and $L_{*}$ galaxies have impact parameters of $\approx 90 \mathrm{kpc}$ and $\approx 260 \mathrm{kpc}$, respectively. From the observed low metallicity, the underabundance of nitrogen, and the lack of $\alpha$-element enhancement the authors argued that the absorber is related to a dwarf galaxy and/or a high velocity cloud in the outskirts of Virgo. The strongest absorber in the sample of Burchett et al. (2017), with $\log N(\mathrm{HI}) / \mathrm{cm}^{-2} \approx 14.9$, also does not have any bright galaxy counterpart within $300 \mathrm{kpc}$ and $|\Delta v|<400 \mathrm{~km} \mathrm{~s}^{-1}$. In this case, the sightline passes through the interface of subclusters A1095W and A1095E. The authors have suggested that stripping from a far away galaxy or a density enhancement due to a merger shockwave are possible origins for the cool gas detected in absorption.

In addition to strong $\mathrm{HI}$, all three systems exhibit strong absorption lines from low-ionization (e.g., C II $\lambda 1036$ ) and intermediate-ionization (e.g., C III $\lambda$ 977, N III $\lambda$ 989) metal lines, suggesting high-metallicity gas. Weak high-ionization lines (e.g., O VI, Ne VIII) might also be present in at least one of them. A detailed analysis of the metal lines, along with ionization models, will be presented in future papers. The analysis of the detected metal lines will provide further insights into the nature of the absorbers.

Support for this research was provided by NASA through grant HST GO-14655 from the Space Telescope Science Institute, which is operated by the Association of Universities for Research in Astronomy, Inc., under NASA contract NAS5-26555. S.M. and J.S. acknowledge support from European Research Council (ERC), grant agreement 278594-GasAroundGalaxies.

\section{ORCID iDs}

Sowgat Muzahid i https://orcid.org/0000-0003-3938-8762 Jane Charlton (i) https://orcid.org/0000-0003-4877-9116 Daisuke Nagai (1) https://orcid.org/0000-0002-6766-5942 Joop Schaye (1) https://orcid.org/0000-0002-0668-5560

\section{References}

Bahé, Y. M., Barnes, D. J., Dalla Vecchia, C., et al. 2017, MNRAS, 470, 4168

Bleem, L. E., Stalder, B., de Haan, T., et al. 2015, ApJS, 216, 27

Burchett, J. N., Tripp, T. M., Wang, Q. D., et al. 2017, MNRAS, submitted (arXiv:1705.05892)

Danforth, C. W., Keeney, B. A., Tilton, E. M., et al. 2016, ApJ, 817, 111

Danforth, C. W., Stocke, J. T., \& Shull, J. M. 2010, ApJ, 710, 613

Gonzalez, A. H., Sivanandam, S., Zabludoff, A. I., \& Zaritsky, D. 2013, ApJ, 778,14

Gonzalez, A. H., Zaritsky, D., \& Zabludoff, A. I. 2007, ApJ, 666, 147

Green, J. C., Froning, C. S., Osterman, S., et al. 2012, ApJ, 744, 60

Hu, W., \& Kravtsov, A. V. 2003, ApJ, 584, 702

Kacprzak, G. G., Muzahid, S., Churchill, C. W., Nielsen, N. M., \& Charlton, J. C. 2015, ApJ, 815, 22

Lau, E. T., Nagai, D., Avestruz, C., Nelson, K., \& Vikhlinin, A. 2015, ApJ, 806,68

Monroe, T. R., Prochaska, J. X., Tejos, N., et al. 2016, AJ, 152, 25

Nagai, D., Lau, E. T., Avestruz, C., Nelson, K., \& Rudd, D. H. 2013, ApJ, 777,137

Osterman, S., Green, J., Froning, C., et al. 2011, Ap\&SS, 335, 257

Prochaska, J. X., Weiner, B., Chen, H.-W., Mulchaey, J., \& Cooksey, K. 2011, ApJ, 740, 91

Prochaska, J. X., Werk, J. K., Worseck, G., et al. 2017, ApJ, 837, 169

Simionescu, A., Allen, S. W., Mantz, A., et al. 2011, Sci, 331, 1576

Tripp, T. M., Jenkins, E. B., Bowen, D. V., et al. 2005, ApJ, 619, 714

Tumlinson, J., Thom, C., Werk, J. K., et al. 2013, ApJ, 777, 59 
Turner, M. L., Schaye, J., Steidel, C. C., Rudie, G. C., \& Strom, A. L. 2014 , MNRAS, 445, 794

Urban, O., Simionescu, A., Werner, N., et al. 2014, MNRAS, 437, 3939

Voit, G. M. 2005, RvMP, 77, 207

Walker, S. A., Fabian, A. C., Sanders, J. S., \& George, M. R. 2012, MNRAS, 427, L45
Werk, J. K., Prochaska, J. X., Tumlinson, J., et al. 2014, ApJ, 792,

Yoon, J. H., \& Putman, M. E. 2013, ApJL, 772, L29

Yoon, J. H., \& Putman, M. E. 2017, ApJ, 839, 117

Yoon, J. H., Putman, M. E., Thom, C., Chen, H.-W., \& Bryan, G. L. 2012, ApJ, 754, 84 